\title{
ANÁLISIS DE ACCIDENTES DE TRABAJO Y ENFERMEDADES PROFESIONALES EN ACTIVIDADES DE PAVIMENTACIÓN CON ASFALTO
}

\section{Orlando Moscote}

Magister en Ciencias Económicas

Universidad Distrital Francisco José de Caldas

oamoscote@hotmail.com

Bogotá, Colombia

\section{Germán Andrés Méndez}

Doctor en Ciencias Técnicas

Universidad Distrital Francisco José de Caldas

gmendez@udistrital.edu.co

Bogotá, Colombia

\section{Carlos Alirio Beltrán Rodríguez}

Magister en Ingeniería Industrial

Universidad Distrital Francisco José de Caldas

cbeltran5@yahoo.es

Bogotá, Colombia

Tipo: Artículo de investigación

Fecha de Recepción: Nov. 15 de 2012

Fecha de Aceptación: Marzo 15 de 2012

\section{AN ANALYSIS ON WORK ACCIDENTS AND PROFESSIONAL ILLNESSES ASSOCIATED TO ASPHALT-ROAD MAKING}

\begin{abstract}
The present paper attempts to determine the main causes of work accidents and professional risks for the workers involved in asphalt road making. The research work was conducted by a research group called SES, who focuses on social problems such as those pertaining to occupational illnesses. The results confirm that there are two major problems associated to this professional activity, namely high probability of having an accident and also of having backaches. Moreover, it is interesting to see the impact of variables like the worker's marital status and his regular sport activities on such accident rates. Regarding the first variable, age appears as a significant correlation aspect (also directly connected with respiratory diseases); regarding the latter variable, it was found that encouraging workers to take up sports activities significantly reduces breathing disorders for workers.
\end{abstract}

Key words: risks, work accident, professional illness, classification trees, logistic model.

\section{RESUMEN}

Dentro de los trabajos desarrollados al interior del grupo de investigación SES, se adelantan esfuerzos por el modelamiento de problemática social, encontrándose como uno de los principales ejes temáticos los riesgos laborales presentes en los trabajadores que realizan la actividad de pavimentación con asfaltos. Por ello, se adelantó una investigación tendiente a determinar las principales causas de accidentalidad en el trabajo y de riesgos profesionales para dichas actividades. De los resultados obtenidos se comprueba dos verdades evidentes y es que la exigencia física del trabajo en la pavimentación genera una alta probabilidad de accidentalidad y una alta incidencia en los dolores de espalda, pero resulta interesante analizar el impacto sobre esta accidentalidad de variables como el impacto del estado civil y la afición a los deportes. En el primer caso se puede explicar por la edad como un correlator tanto de la edad como de las enfermedades respiratorias y en el segundo caso es mejor estimular la práctica de los deportes para evitar los 
problemas respiratorios en este tipo de industria.

Palabras clave: riesgos, accidente de trabajo, enfermedad profesional, arboles de clasificación, modelo logístico.

\section{INTRODUCCIÓN}

La situación de la salud ocupacional en el sector de la construcción vial en Colombia y especialmente en Bogotá en donde prácticamente en las dos últimas décadas la ciudad está en construcción y reconstrucción de su malla vial para acomodarse a los requisitos de sistemas masivos de transporte al igual que al continuo crecimiento urbano, reviste una gran complejidad, ya que a pesar de no disponer de cifras confiables, son evidentes muchas de las deficiencias en este sector que por lo general se traducen en un alto número de lesiones, muertes y deterioro de las condiciones de la salud en relación con el trabajo.

Por lo tanto en este articulo se presentan los resultados del análisis de las variables que tienen una mayor incidencia en la ocurrencia de accidentes de trabajo entendido como todo suceso repentino que sobrevenga por causa o con ocasión del trabajo, y que produzca en el trabajador una lesión orgánica, una perturbación funcional, una invalidez o la muerte [1] y enfermedades profesionales analizadas como todo estado patológico permanente o temporal que sobrevenga como consecuencia obligada y directa de la clase de trabajo que desempeña el trabajador, o del medio en que se ha visto obligado a trabajar, y que haya sido catalogada como enfermedad profesional por el gobierno nacional [2] en los trabajadores de este sector en la ciudad de Bogotá, en donde a partir de herramientas estadísticas como los modelos de respuesta cualitativa (modelo logístico y árboles de clasificación) se evaluaron aspectos como las características de la población trabajadora en el sector, los antecedentes de salud y la accidentalidad.
La enciclopedia de salud y seguridad en el trabajo en su volumen numero 3 capitulo 93 referente al sector de la construcción define los asfaltos como complejas mezclas de componentes químicos de alto peso molecular, predominantemente asfáltenos, hidrocarburos cíclicos (aromáticos o nafténicos) y una cantidad menor de componentes saturados de baja reactividad química. La composición química de los asfaltos depende tanto del petróleo crudo original como del proceso utilizado durante el refino. Entre la variedad de aplicaciones que tiene el asfalto se incluye la pavimentación de calles, carreteras y aeropuertos, materiales para cubiertas, impermeabilización y aislamiento, revestimiento de canales y depósitos de riego; y también el revestimiento de presas y diques. Se estima que la producción anual de asfalto supera actualmente en todo el mundo los 60 millones de toneladas, de las cuales más del $80 \%$ se emplean para las necesidades de construcción y mantenimiento.

Se han realizado mediciones de la exposición a las partículas de hidrocarburos aromáticos polinucleares (PAH) en diferentes ambientes. La mayoría de los PAH detectados estaban compuestos de derivados de naftaleno el cual representa una gran probabilidad de riesgo carcinógeno significativo. Existe escasa información referente a exposiciones a los humos de asfalto en otras situaciones industriales o durante la aplicación o utilización de los productos asfálticos. El manejo del asfalto caliente puede causar graves quemaduras, debido a que es pegajoso y no se quita fácilmente de la piel. La principal preocupación, desde el punto de vista toxicológico industrial, es la irritación de la piel y de los ojos por los humos del asfalto caliente. Estos humos pueden causar dermatitis y lesiones parecidas al acné, así como queratosis ligera en caso de exposiciones 
repetidas y prolongadas.

Los efectos sobre el árbol traqueo bronquial y los pulmones de los ratones al inhalar un aerosol de asfalto y, en otro grupo que inhaló humo de asfalto calentado, dieron lugar a congestión, bronquitis, neumonía, dilatación bronquial, cierta infiltración en las células redondas peri bronquiales, formación de abscesos, pérdida ciliar, atrofia epitelial y necrosis. Se llegó a la conclusión de que estos cambios constituían un fenómeno general causado por respirar aire contaminado con hidrocarburos aromáticos, y que el grado de cambio depende de la dosis respirada. Se han realizado pruebas sobre los efectos de la aplicación de asfaltos refinados al vapor a la piel de los ratones. Los asfaltos no disueltos, las disoluciones en benceno y una fracción de asfalto refinado al vapor produjeron tumores de la piel. Una mezcla de asfaltos destilados al aire y al vapor en benceno produjo tumores en los puntos de aplicación de la piel de ratones. Una muestra de asfaltos refinados al aire calentado, inyectada subcutáneamente en los ratones, produjo algunos sarcomas en los puntos de inyección. Un extracto de asfalto del pavimento de una carretera y sus emisiones tuvieron efectos mutágenos en la salmonella typhimurium.

No existen pruebas concluyentes de su carcinogénesis en las personas. Un grupo numeroso de trabajadores de cubiertas expuestos a betunes tanto asfálticos como de alquitrán mostró un riesgo superior de cáncer respiratorio. Asimismo, dos estudios daneses revelan un riesgo superior de cáncer de pulmón, pero algunos de los trabajadores habían estado expuestos también al alquitrán y, probablemente, eran más adictos al tabaco que el resto del grupo.

Entre los trabajadores en carreteras en Minnesota (pero no en California) se apreciaron incrementos de cánceres urológicos y leucemia. Aunque los datos epidemiológicos de que se dispone hasta la fecha no bastan para demostrar con un grado razonable de certeza científica que el asfalto represente un riesgo de cáncer para las personas, existe un consenso generalizado de que, a la luz de estudios experimentales, tal riesgo es posible. Los extendedores de asfalto y el conductor de una máquina extendedora deben llevar máscaras de respiración de media cara con cartuchos para vapores orgánicos. Además, para evitar la ingestión involuntaria de materiales tóxicos, los trabajadores no deben comer, beber o fumar junto a una caldera [3].

La IARC (international agency for research on cáncer) clasifica los betunes/asfaltos en [4]:

Clase 1: Los betunes de penetración son el producto residual de la destilación atmosférica del petróleo crudo aplicando una destilación al vacío, una oxidación parcial, una precipitación por disolventes o una combinación de dichos procesos.

Clase 2: Los betunes oxidados se clasifican por su punto de reblandecimiento y grado de penetración.

Clase 3: Los betunes fluidificados se producen mezclando betunes de penetración y betunes oxidados con disolventes volátiles adecuados procedentes del petróleo crudo.

Clase 4: Los betunes duros se clasifican normalmente por su punto de reblandecimiento y se fabrican de manera similar a los betunes de penetración.

Clase 5: Las emulsiones bituminosas son finas dispersiones de gotas de betún (de las clases 1 , 3 o 6) en agua. Se fabrican usando batidoras de alta velocidad, como los molinos coloidales.

Clase 6: Los betunes mezclados o fluxados pueden fabricarse mezclando betunes con extractos de disolventes con residuos termofisurados del petróleo pesado, o con ciertos productos destilados del petróleo pesado con un punto de ebullición final superior a $350{ }^{\circ} \mathrm{C}$.

Clase 7: Los betunes modificados contienen cantidades apreciables (normalmente del 3 al $15 \%$ en peso) de aditivos especiales, 
como polímeros, elastómeros, sulfuros y otros productos usados para modificar sus propiedades; se emplean para aplicaciones especiales.

Clase 8: Los betunes térmicos se fabricaban por destilación prolongada a altas temperaturas de residuos del petróleo.

\section{VALORACIÓN CUALITATIVA Y CUANTITATIVA DE LOS FACTORES DE RIESGO}

Se realizó una evaluación y priorización de los factores de riesgos basados en la metodología propuesta en la guía técnica colombiana GTC - 45, con base en la información recopilada a través del panorama de riesgos. Para este caso se avaluaron las tres actividades que se realizan durante la aplicación del asfalto en la pavimentación de vías las cuales incluyen nivelación, imprimación y pavimentación, a partir de las cuales se identificaron los factores de riesgos asociados al proceso constructivo como riesgos físicos (ruido, radiaciones, vibraciones, temperaturas), químicos (polvos, gases y vapores), ergonómicos (manejo de cargas, posturasinadecuadas), biológicos (virus, bacterias, animales), mecánicos (manuales, mecanizados), locativos (condiciones de la superficie de trabajo) los cuales pueden generar accidentes de trabajo y enfermedades profesionales para una población de 985 trabajadores.

\subsection{Priorización de riesgos}

El panorama de riesgos permito establecer la priorización de los factores de riesgo con un alto grado de peligrosidad y repercusión, los cuales son descritos a continuación:

Priorización de riesgos - nivelación y compactación: Este trabajo consiste en la instalación de material de base granular aprobado, sobre una superficie preparada, en una o varias capas, de conformidad con los alineamientos, pendientes y dimensiones indicados en los planos del proyecto (tabla 1).
Tabla 1. Priorización de riesgos - nivelación y compactación.

\begin{tabular}{|c|l|}
\hline \multicolumn{2}{|c|}{ FACTOR DE RIESGO } \\
\hline Físicos & Energía mecánica (ruido) \\
\hline \multirow{2}{*}{ Químicos } & $\begin{array}{l}\text { Sólidos: Concentración de material } \\
\text { particulado }\end{array}$ \\
\cline { 2 - 2 } & Gases: (CO,CO2,SO2,NO2) \\
\hline \multirow{2}{*}{ Ergonómicos } & $\begin{array}{l}\text { Carga dinámica } \\
\text { (sobreesfuerzos y movimientos) }\end{array}$ \\
\cline { 2 - 2 } & Carga estática (de pié o sentado) \\
\hline
\end{tabular}

Priorización de riesgos - imprimación: este paso comprende el retiro de cualquier material suelto o basura de la superficie a reparar, una tarea en la que el uso de aire comprimido da buenos resultados. Si se llega hasta la base o sub-base perturbando los materiales que la constituyen, es necesario compactarlos, para evitar que se produzcan asentamientos por efectos del tránsito. Si la causa de la falla es el agua se deben instalar drenajes. La presencia de agua genera una mala adhesión del parche, por lo que la superficie a reparar se debe secar con aire comprimido. Luego debe aplicarse una capa ligante, con el equipo adecuado, una capa de pega en las caras verticales, para establecer una buena adherencia entre las superficies vieja y nueva, evitando el exceso de ligante (tabla 2).

Tabla 2. Priorización de riesgos - imprimación.

\begin{tabular}{|c|l|}
\hline \multicolumn{2}{|c|}{ FACTOR DE RIESGO } \\
\hline Físicos & Energía mecánica (ruido) \\
\hline \multirow{2}{*}{ Químicos } & Líquidos: Emulsión asfáltica \\
\cline { 2 - 2 } & Gases: (CO,CO2,SO2,NO2) \\
\hline Ergonómicos & Carga estática (de pié) \\
\hline
\end{tabular}

Colocación de pavimento (asfalto): la mezcla asfáltica al ser descargada en la máquina pavimentadora debe tener una temperatura que permita obtener las densidades de campo establecidas, pero nunca debe ser menor de $105^{\circ} \mathrm{C}$. El extendido de la mezcla asfáltica se debe hacer por medio de máquinas pavimentadoras, sin que se produzcan arrastres o desgarramientos de la capa que se está extendiendo. En caso de que se presenten 
áreas defectuosas, estas deben ser corregidas utilizando los procedimientos que sean aprobados por la inspección de la obra (tabla $3)$.

Tabla 3. Colocación de pavimento (asfalto).

\begin{tabular}{|c|l|}
\hline \multicolumn{2}{|c|}{ FACTOR DE RIESGO } \\
\hline Físicos & Energía mecánica (ruido) \\
\hline Químicos & Gases: (CO,CO2,SO2,NO2) \\
\hline \multirow{3}{*}{ Ergonómicos } & $\begin{array}{l}\text { Carga Dinámica } \\
\text { (sobreesfuerzos y movimientos) }\end{array}$ \\
\cline { 2 - 2 } & Carga estática (de pié o sentado) \\
\hline
\end{tabular}

\section{CONTEXTUALIZACIÓN Y DISEÑO DEL INSTRUMENTO DE PARA LA RECOLECCIÓN DE DATOS}

Al realizar un muestreo probabilístico uno de los principales aspectos que más le interesa al investigador es ¿Cuál es el número mínimo de unidades de análisis que se necesitan para conformar una muestra $(n)$ ? Para este caso la población de interés son las obras de pavimentación con asfalto que se desarrollan en Bogotá [5].

\subsection{Información general}

Como información general se estableció en primer lugar las características del trabajo en donde se tuvo en cuenta el cargo, las funciones que desarrolla, el tiempo en la empresa y en el cargo. En segundo lugar se definieron características del trabajador como la edad, el género (sexo), la escolaridad y el estado civil. Finalmente, se definieron las relaciones interpersonales, en donde se le preguntó a cada trabajador cómo son sus relaciones con las personas que convive, con el cónyuge, con los hijos y con los compañeros de trabajo.

\subsection{Antecedentes de salud [6]}

En una alianza con los EE.UU. Administración federal de carreteras, NIOSH llevó a cabo un estudio para desarrollar y probar nuevos métodos de campo para evaluar la exposición las emanaciones del asfalto, caracterizar y comparar la exposición ocupacional a la miga de goma asfalto modificado (CRM) y el "asfalto convencional", y evaluar los efectos potenciales para la salud de la exposición (Burr et al., 2002). Para cada sitio, el asfalto convencional tuvo la misma formulación que el CRM, pero no contiene la miga de goma. Este CICAD contiene sólo la información del análisis de siete sitios de asfalto convencional de pavimentación. Dos grupos de trabajadores (expuestos y no expuestos) fueron reclutados en cada sitio, las evaluaciones médicas se llevaron a cabo durante 4 días - 2 días para CRM y 2 días para que el asfalto convencional. Un cuestionario de salud general fue completado por cada participante en el inicio de un estudio del sitio, preguntando acerca de la historia reciente de los ojos, nariz o garganta irritada, tos, dificultad para respirar, sibilancias, y la historia de los trastornos respiratorios crónicos. Antecedentes de fumar y el trabajo también se han obtenido. Un cuestionario de abordar los síntomas agudos se distribuyó a los trabajadores antes y postshift, 3 veces durante la jornada de trabajo. Tasa de flujo espiratorio máximo se midió justo antes de completar el cuestionario de síntomas agudos. Vigilancia de la zona y PBZ se llevaron a cabo para evaluar la exposición en el aire. Un total de 94 trabajadores empleados en cualquiera de los siete sitios de pavimentación participaron en el estudio. Los resultados se presentan en la siguiente sección.

Los efectos respiratorios: Entre las poblaciones de los trabajadores, los efectos agudos de la exposición a las emanaciones de asfalto son los síntomas de irritación de las membranas serosas de las conjuntivas (irritación de los ojos) y las membranas mucosas del tracto respiratorio superior (irritación nasal y de garganta). Síntomas similares también han sido reportados en los trabajadores expuestos a los humos de asfalto en la aplicación de material para techos de asfalto caliente (Exxon, 1997), durante la fabricación de tejas asfálticas (Apol y Okawa, 1977, Exxon, 1997), y en mezcla en caliente plantas de asfalto y los terminales (Exxon, 1997). En un estudio de cinco situaciones diferentes de asfalto de la exposición (en caliente mezclar 
plantas, terminales, aplicaciones para techos, fabricación de techos y pavimentos), aunque los síntomas fueron reportados, no significativa dosis-respuesta se encontró una asociación entre la exposición medida y los síntomas (Exxon, 1997).

Sin embargo, en el estudio de NIOSH de pavimentadoras de asfalto convencional, las concentraciones en aire de TP, BSP, y las PAC fue significativamente mayor en los días cuando los síntomas del ojo, la nariz o la garganta se compararon con los presentes días en que los síntomas no se informaron ( $\mathrm{P}=0,02$; $\mathrm{P}<0,01$ y $\mathrm{P}<0,01$, respectivamente) (Burr et al., 2002). Mientras que las concentraciones de las emanaciones del asfalto asociados con los efectos de salud se señaló anteriormente, no han sido bien caracterizados, los síntomas de irritación de los ojos, la nariz o la garganta fueron reportados por los trabajadores durante al aire libre de pavimentación. Medio, la exposición personal, calculado como TWA turno completo, en general fueron inferiores a $1,0 \mathrm{mg} / \mathrm{m}^{3}$ de TP y $0,3 \mathrm{mg} / \mathrm{m}^{3}$ para el BSP (Almaguer et al, 1996; . Hanley \& Miller, 1996a, b;. Kinnes et al, 1996; Miller y Burr, 1996a, b, 1998; Exxon, 1997).

Algunos trabajadores con experiencia menor problemas de las vías respiratorias o cambios en la función pulmonar cuando son expuestos a TP en concentraciones que oscilan entre 0,02 y $1 \mathrm{mg} / \mathrm{m}^{3}$ en aire libre, carretera asfaltada (Almaguer et al, 1996; . Hanley \& Miller, 1996a, b; Kinnes et al, 1996;. Miller y Burr, 1996a, b, 1998; Exxon, 1997; Gamble et al, 1999).. Kinnes et al. (1996) reportaron cambios significativos en la función pulmonar en uno de los siete trabajadores que realizan al aire libre de pavimentación asfáltica. En tres de los nueve trabajadores contratados en el metro de pavimento, bronchoreactivity aumento se observó, aunque sólo una extendedora informaron de síntomas (Sylvain y Miller, 1996). Las concentraciones de TP varió desde 1,09 hasta $2,17 \mathrm{mg} / \mathrm{m}^{3}$ y la concentración de BSP varió desde 0,3 hasta $1,26 \mathrm{mg} / \mathrm{m}^{3}$ durante subterráneo pavimentación (Sylvain y Miller, 1996). El estudio de los trabajadores en cinco segmentos de la industria de asfalto (Exxon, 1997;. Gamble et al, 1999) no encontró ninguna asociación significativa entre las mediciones de función pulmonar (FVC, FEV1, FEF y 2575 durante un turno laboral) y la exposición al asfalto entre los trabajadores. Alguna evidencia limitada sugiere que los factores personales de salud (por ejemplo, asma pre-existente) o la exposición a mayores cantidades de gases del asfalto, como las que se encuentran en tierra de pavimentación, pueden aumentar el riesgo de los trabajadores para los síntomas de las vías respiratorias o cambios en la función pulmonar (Norseth et al, 1991;. Sylvain y Miller, 1996). Sin embargo, los datos actuales son insuficientes para determinar la relación entre la exposición a las emanaciones del asfalto y los efectos sobre la salud.

Además, otros riesgos de confusión potenciales, como la de escape de gasolina y diesel y el camino y el polvo de los neumáticos, también puede contribuir con un potencial aún sin cuantificar para la irritación de las vías respiratorias.

Bronquitis aguda y crónica, posiblemente relacionado con la irritación crónica del tracto respiratorio inferior, se han reportado entre los trabajadores del asfalto en varios estudios (Zeglio, 1950; Baylor y Weaver, 1968; Hasle et al, 1977;. Nyqvist, 1978;. Maintz et al, 1987; Hansen, 1991). Irritación de la piel, prurito o erupciones también se informó después de la exposición a los materiales a base de asfalto (Tavris et al, 1984; . Schaffer et al, 1985; . Waage y Nielson, 1986; Chase et al, 1994;. Miller y Burr, 1996a , b, 1998). Teniendo en cuenta la presencia de confusión co-exposición (es decir, los productos de escape de combustible diesel, alquitrán de hulla, fibra de vidrio) y condiciones ambientales (viento, el calor y la humedad, la radiación ultravioleta), en la medida en que las emanaciones de asfalto que pueden estar asociados con estos problemas de la piel no es clara.

Los síntomas de dolor de estómago, náuseas, disminución del apetito, dolores de cabeza y fatiga también se han reportado entre los 
trabajadores expuestos al asfalto (Tavris et al, 1984;. Schaffer et al, 1985;. Waage y Nielson, 1986; . Norseth et al, 1991 , Chase et al, 1994;. Exxon, 1997; Gamble et al, 1999).. Con la excepción de los estudios de Norseth et al. (1991), Exxon (1997), and Gamble et al. (1999), ninguno de los otros informes examinaron los grupos de comparación que permite analizar los efectos de exposiciónrespuesta. Norseth et al. (1991) encontró una mayor frecuencia de apetito y la reducción de la fatiga de los trabajadores expuestos al asfalto en comparación con los controles.

No significativa dosis-respuesta se encontró una asociación entre la exposición medida y los síntomas en cualquiera de las cinco situaciones de asfalto de la exposición (Exxon, 1997; Gamble et al, 1999).. La medida en que los gases del asfalto puede estar asociada con los síntomas anteriores está claro, teniendo en cuenta posibles factores de confusión.

El cáncer de pulmón entre los adoquines: mientras que varios estudios han reportado un riesgo elevado de cáncer de pulmón (Hansen, 1989, 1991; Engholm et al, 1991; Partanen et al, 1997), limitaciones en el diseño de algunos de estos estudios impiden sacar conclusiones fuertes.

En este apartado de la encuesta se definieron hábitos como el tabaquismo, el alcoholismo y la práctica de algún deporte, preguntando a cada trabajador si fuma, si ingiere alcohol y si practica algún deporte y su respectiva frecuencia, con el propósito de establecer el comportamiento de los trabajadores frente a estos hábitos.

Por otro lado y a partir de los posibles efectos de la exposición a gases por respirar los vapores del asfalto, como dolores de cabeza, erupciones de la piel, fatiga, irritación de los ojos y de la garganta y tos, que dependen, entre otras cosas, de la intensidad y de la duración de la exposición, se definieron las posibles enfermedades debidamente diagnosticadas por un médico entre las cuales se tienen: Tensión alta, problemas auditivos, dolor de espalda, problemas de colesterol, problemas gástricos, alteraciones visuales, problemas cardiacos, problemas respiratorios y problemas de la piel.

Finalmente, se definieron síntomas que se pueden presentar por la exposición a las emanaciones de asfalto y la frecuencia con la cual se presentan (No, rara vez, ocasional o frecuentemente) como: Dolor de cabeza, tos, ronquera, cansancio visual, disminución de la audición, secreción nasal, dolor de espalda, dolor o molestias en las manos, insomnio, disminución en la concentración, problemas de memoria, ardor en el estómago, irritabilidad, ansiedad y depresión. Se estableció a partir de la frecuencia de los síntomas, si estos están asociados con el trabajo o no, definiendo si se presentan antes, durante, después o no tienen ninguna relación con el trabajo.

\subsection{Accidentalidad [7]}

Los accidentes más comunes al trabajar con el asfalto son las quemaduras producidas al contacto del material caliente con la piel. En caso de ocurrencia de una quemadura, se recomienda seguir los siguientes pasos: Sumergir la parte afectada en agua fría, por al menos 10 minutos. Si se tiene hielo, no aplicarlo directamente en el área afectada, sino usarlo para enfriar el agua. Solicitar atención médica. En caso que la quemadura ocurra en la cabeza o cara, o involucre una gran superficie, se debe recurrir a un centro médico de inmediato. Por ningún motivo se debe intentar remover el asfalto de la piel, una vez que éste se enfríe no producirá más daño y servirá como protección del área afectada. A medida que la herida sane el asfalto se desprenderá, normalmente dentro de 2 a 3 días. Si es necesario remover el asfalto de la piel se puede usar parafina médica u otro solvente médicamente aceptado, ya que otros solventes pueden causar más daño. Posteriormente se debe lavar el área quemada con agua y jabón.

Otro problema común es la inhalación prolongada de gases provenientes del asfalto, principalmente durante la fabricación y 
colocación de mezcla asfáltica. Los efectos que puede producir la exposición frecuente a estos gases son: dolor de cabeza, erupciones en la piel, fatiga, reducción del apetito, irritación de ojos y garganta, y tos. Estos problemas se pueden producir con dosis superiores a $0.5 \mathrm{mg} / \mathrm{m}^{3}$, el cual es el límite de exposición que recomienda la OSHA (occupational safety and health administration, de los Estados Unidos). En caso de inhalación prolongada, se recomienda llevar al afectado a un área ventilada. Si los síntomas permanecen o se intensifican, administrar oxígeno al afectado y solicitar atención médica.

El manejo del asfalto cortado no presenta peligro de quemaduras, sin embargo existen riesgos como las reacciones alérgicas al contacto con la piel: Para evitarlas se debe usar ropa y elementos protectores y seguir prácticas habituales de higiene y aseo personal. Salpicaduras en los ojos: Se recomienda que el personal expuesto use anteojos de protección. Ingestión accidental: En el caso de contaminación oral simple, se debe enjuagar la boca con abundante agua. La ingestión del producto requiere atención médica. Como las emulsiones se manejan a temperaturas inferiores a $85^{\circ} \mathrm{C}$, no presentan peligro de quemaduras, sin embargo existe el riesgo de reacciones alérgicas al contacto con la piel: Para evitarlas se debe usar ropa y elementos protectores y seguir prácticas habituales de higiene y aseo personal. Salpicaduras en los ojos: Se recomienda que el personal expuesto use anteojos de protección. Ingestión accidental: En el caso de contaminación oral simple, se debe enjuagar la boca con abundante agua. La ingestión del producto requiere atención médica.

Las características de la accidentalidad se definieron a partir de los formatos de reporte de accidentes de trabajo que aplican las administradoras de riesgos profesionales en Colombia para registrar estos eventos; con esta información y con ayuda del instrumento de recolección de datos se incluyo la parte del cuerpo afectada como la cabeza, extremidades superiores, tórax y extremidades inferiores; y por otro lado, se tuvo en cuenta el tipo de lesión o daño aparente entre los cuales se tienen: Quemadura calórica, quemadura química, esguince o torcedura, reacción alérgica, efecto radiación ionizante, efecto radiación ionizante, raspadura, herida, luxación, insolación, hernias, politraumatismo, intoxicación, desgarro, golpe o trauma, fractura, amputación, congelación, contusión cerebral y pérdida de audición.

\section{APLICACIÓN Y LEVANTAMIENTO DE LA INFORMACIÓN}

Con el diseño del instrumento para recolección de datos (encuesta) se procedió a establecer una muestra representativa de trabajadores del sector de la construcción específicamente de las obras donde se realiza pavimentación de vías con asfalto con el propósito de evaluar aspectos ocupacionales y condiciones de trabajo.

La población de interés son obras de pavimentación que se realizan en Bogotá D.C., las cuales son desarrolladas por el Instituto de desarrollo urbano (IDU), la unidad especializada de mantenimiento de la malla vial y las empresas de servicios públicos. Por lo tanto, en este se incluyen todas las obras de infraestructura que son ejecutadas en la ciudad, (ver tabla 4).

Con el diseño del instrumento para recolección de datos (Encuesta) se procedió a establecer una muestra representativa de trabajadores del sector de la construcción específicamente de las obras donde se realiza pavimentación de vías con asfalto con el propósito de evaluar aspectos ocupacionales y condiciones de trabajo.

La población de interés son obras de pavimentación que se realizan en Bogotá D.C., las cuales son desarrolladas por el Instituto de desarrollo urbano (IDU), la unidad especializada de mantenimiento de la malla vial y las empresas de servicios públicos. Por lo tanto, en este se incluyen todas las obras de infraestructura que son ejecutadas en la ciudad, (ver tabla 4). 
Tabla 4. Contratos vigentes de Febrero a Noviembre de 2009. Fuente: Secretaria distrital de movilidad 2009.

\begin{tabular}{|l|c|}
\hline \multicolumn{1}{|c|}{ TIPO DE CONTRATO } & CANTIDAD \\
\hline Obras de infraestructura & 220 \\
\hline Acciones de movilidad & 33 \\
\hline Diagnostico & 26 \\
\hline Demarcación & 69 \\
\hline TOTAL CONTRATOS & 348 \\
\hline
\end{tabular}

Durante el periodo comprendido entre Febrero a Noviembre de 2009 se tuvo en cuenta 348 intervenciones aprobadas por el comité de obras de infraestructura discriminadas por construcción de vías en concreto, construcción de espacio público, actividades de diagnostico de vías, actividades de demarcación de vías y pavimentación de vías con asfalto. De esto se requirió de manera previa realizar un filtrado de la información por intervenciones en las cuales se realizaran pavimentación de vías con asfalto la cual arrojó como resultado que de las 348 intervenciones, 120 consisten en obras de infraestructura con pavimentación de vías en asfalto. Para realizar este análisis se calculó el tamaño de la muestra a partir de la Ec. (1).

$$
n=\frac{N Z^{2} P(1-P)}{(N-1) e^{2}+Z^{2} P(1-P)}
$$

Donde:

$N$ : Tamaño de la población.

$Z$ : Valor en la distribución normal estándar.

e: Máximo error deseable.

P: Proporción de elementos que se supone tienen la característica deseada en la población.

Considerando:

- Nivel de confianza: 0.95 , por lo cual $\mathrm{Z}=1.96$

- Máximo error permisible: e=0.06

- Número de obras de pavimentación: N=120

- Proporción de obras en donde han ocurrido accidentes de trabajo: $\mathrm{P}=0.5$

- Tamaño de muestra: $\mathrm{n}=36$

Al aplicar la formula anterior se seleccionaron 36 contratos de obra.

\section{RESULTADOS}

Una vez se seleccionaron los 36 contratos de obra y en los cuales se encuestó al total de la población trabajadora que corresponden a 985 trabajadores durante Febrero a Noviembre del año 2009, se presentan los principales resultados obtenidos a partir del análisis con modelos de respuesta cualitativa como:

El modelo logístico [8]: esta técnica se aplica a situaciones donde las variables explicativas no tienen una distribución conjunta normal multivariante, en donde se espera que el modelo se comporte bien cuando todas las variables de clasificación son binarias y aproximadamente independientes Que como se verá más adelante es el caso bajo objeto de estudio.

Arboles de clasificación [9]: el procedimiento de clasificación basado en árboles permite ordenar los casos en grupos o pronosticar valores de una variable (criterio) dependiente basada en valores de variables independientes (predictores). El procedimiento proporciona herramientas de validación para análisis de clasificación exploratorios y confirmatorios. Un análisis basado en árboles ofrece algunas características atractivas ya que:

- Permite identificar grupos homogéneos con alto o bajo riesgo.

- Facilita la construcción de reglas para realizar pronósticos sobre casos individuales.

\subsection{Análisis de accidentes de trabajo}

El análisis de los accidentes de trabajo se realizó mediante los métodos de regresión logística y árboles de clasificación tomando como variable dependiente los accidentes de trabajo y como variables independientes: Características del trabajo (cargo y tiempo en el cargo en meses), características del trabajador (Sexo, edad, estado civil y escolaridad), relaciones interpersonales (con las personas que convive, con el cónyuge, con los hijos y con los compañeros de trabajo) y hábitos (Fumar, ingesta de alcohol, practica de algún deporte y 
su frecuencia). Se reunieron las variables que tuvieron mayor incidencia en los accidentes de trabajo obteniendo los siguientes resultados (todos los valores fueron obtenidos mediante el programa SPSS [10])):

\section{A. Análisis con regresión logística para accidentes de trabajo}

Considerando un nivel de significancia desde 0,1 las variable que resultan ser significativas son las indicadas con * (ver tabla 5).

Tabla 5. Variables en la ecuación.

Fuente: SPSS

\begin{tabular}{|l|c|c|c|c|c|c|}
\hline & B & E.T. & Wald & gl & Sig. & Exp(B) \\
\hline Deporte* $^{*} .649$ & .152 & 18.3 & 1 & .000 & 1.913 \\
\hline Fuma* & .436 & .141 & 9.60 & 1 & .002 & 1.546 \\
\hline Escolaridad* & -.560 & .220 & 6.47 & 1 & .011 & .571 \\
\hline $\begin{array}{l}\text { Relación } \\
\text { hijos* }\end{array}$ & .306 & .129 & 5.58 & 1 & .018 & 1.358 \\
\hline $\begin{array}{l}\text { Relación } \\
\text { cónyuge }\end{array}$ & -.104 & .121 & .746 & 1 & .388 & .901 \\
\hline Deporte* & .649 & .152 & 18.3 & 1 & .000 & 1.913 \\
\hline Cargo & .249 & .221 & 1.27 & 1 & .259 & 1.283 \\
\hline
\end{tabular}

B. Análisis con árboles de clasificación para accidentes de trabajo

Al realizar el árbol de clasificación con estas variables el resultado indica que la escolaridad, la práctica de algún deporte y si fuman son las variables que más incidencia tienen sobre la accidentalidad.

\subsection{Análisis enfermedades diagnosticadas}

El análisis de las enfermedades diagnosticadas se estableció en función de los accidentes ocurridos, según las enfermedades diagnosticadas por parte de un médico y si estos trabajadores habían sufrido accidentes de trabajo, por lo tanto se obtuvieron los siguientes resultados:

\subsubsection{Análisis para problemas de espalda}

Se reunieron las variables que tuvieron una mayor incidencia en los problemas de espalda, de acuerdo con los resultados obtenidos mediante los métodos árboles de clasificación y regresión logística.

\section{A. Análisis con árboles de clasificación para problemas de espalda}

Al realizar el árbol de clasificación se observa que en los problemas de espalda las variables que tienen una mayor incidencia son el dolor de espalda, el cargo, la depresión, el dolor en las manos y la disminución de la audición. Debido a que la comodidad es una respuesta individual, es bastante difícil definir principios estrictos de postura [11].

En donde $\mathrm{B}$ es el coeficiente de la variable Accidente, E.T. es su error estándar, Wald es el estadístico del contraste de hipótesis (siendo Ho: $B=0$ ), gl son los grados de libertad, sig. es el valor $p$ asociado al contraste y EXP (B) corresponde a la OR de la variable analizada (accidentes de trabajo) (tabla 6 y 7 ).

Tabla 6. Variables en la ecuación - dolor de espalda. Fuente: SPSS

\begin{tabular}{|l|c|c|c|c|c|c|}
\hline & $\mathrm{B}$ & E.T. & Wald & gl & Sig. & Exp(B) \\
\hline $\begin{array}{l}\text { Dolor de } \\
\text { espalda* }\end{array}$ & .468 & .078 & 35.742 & 1 & .000 & 1.596 \\
\hline $\begin{array}{l}\text { Dolor de } \\
\text { manos* }\end{array}$ & .303 & .087 & 12.237 & 1 & .000 & 1.354 \\
\hline Depresión* & .473 & .148 & 10.187 & 1 & .001 & .623 \\
\hline Cargo* & .700 & .230 & 9.285 & 1 & .002 & .497 \\
\hline $\begin{array}{l}\text { Disminución } \\
\text { de audición }\end{array}$ & -.032 & .096 & .107 & 1 & .744 & .969 \\
\hline
\end{tabular}

Tabla 7. Clasificación Problemas de espalda. Fuente: SPSS

\begin{tabular}{|c|c|c|c|}
\hline \multirow{2}{*}{ Observado } & \multicolumn{3}{|c|}{ Pronosticado } \\
\cline { 2 - 4 } & No & $\mathrm{Si}$ & $\begin{array}{c}\text { Porcentaje } \\
\text { correcto }\end{array}$ \\
\hline $\mathrm{No}$ & 577 & 43 & $93.1 \%$ \\
\hline $\mathrm{Si}$ & 270 & 95 & $26.0 \%$ \\
\hline $\begin{array}{c}\text { Porcentaje } \\
\text { global }\end{array}$ & $86.0 \%$ & $14.0 \%$ & $68.2 \%$ \\
\hline
\end{tabular}

B. Análisis con regresión logística para problemas de espalda

Al realizar la regresión logística se observa que en los problemas de espalda las variables que 
tienen una mayor incidencia son el dolor de espalda, el dolor en las manos, la depresión y el cargo (tabla 8).

Tabla 8. Tabla de clasificación problemas de espalda. Fuente: SPSS

\begin{tabular}{|c|c|c|c|}
\hline \multirow{3}{*}{ Observado } & \multicolumn{3}{|c|}{ Pronosticado } \\
\hline & \multicolumn{2}{|c|}{ Dolor de espalda } & \multirow{2}{*}{$\begin{array}{c}\% \\
\text { Correcto }\end{array}$} \\
\hline & No & $\mathrm{Si}$ & \\
\hline No & 538 & 82 & 86.8 \\
\hline $\mathrm{Si}$ & 248 & 117 & 32.1 \\
\hline$\%$ Global & & & 66.5 \\
\hline
\end{tabular}

\subsubsection{Análisis para problemas auditivos}

Se reunieron las variables que tuvieron una mayor incidencia en los problemas auditivos, de acuerdo con los resultados obtenidos mediante los métodos árboles de clasificación y regresión logística.

\section{A. Análisis con árboles de clasificación para problemas auditivos}

Al realizar el árbol de clasificación se observa que en los problemas auditivos las variables que tienen una mayor incidencia son las relaciones con los compañeros de trabajo, los problemas de memoria, la disminución de la audición y el dolor de cabeza.

Esto confirma lo que dicen los diferentes estudios acerca de los efectos del ruido en la salud, ya que este afecta física y psicológicamente al organismo, ya que está demostrado que cuando los ruidos producen más de 60 decibeles se puede llegar a presentar aceleración de la respiración y del pulso, aumento de la presión arterial, problemas digestivos que ocasionan gastritis, problemas neuromusculares que ocasionan dolor y falta de coordinación, disminución de la visión nocturna, aumento de la fatiga y dificultad para dormir, disminución de la concentración, la efectividad y la productividad y aumento de la frecuencia de accidentes de trabajo y la irritabilidad. La exposición a niveles de ruido moderado y alto corresponde con un aumento de la fatiga [12] (tabla 9 y 10).
Tabla 9. Variables en la ecuación - Problemas auditivos. Fuente: SPSS

\begin{tabular}{|l|c|c|c|c|c|c|}
\hline & B & E.T. & Wald & gl & Sig. & Exp(B) \\
\hline $\begin{array}{l}\text { Relación } \\
\text { compañeros } \\
\text { trabajo* }\end{array}$ & -.511 & .156 & 10.75 & 1 & .001 & .600 \\
\hline Depresión* & -.347 & .127 & 7.522 & 1 & .006 & .707 \\
\hline $\begin{array}{l}\text { Disminución } \\
\text { audición* }\end{array}$ & .182 & .074 & 6.013 & 1 & .014 & 1.199 \\
\hline $\begin{array}{l}\text { Dolor de } \\
\text { cabeza }\end{array}$ & -.016 & .074 & .045 & 1 & .833 & .984 \\
\hline $\begin{array}{l}\text { Pérdida de } \\
\text { memoria* }\end{array}$ & .225 & .119 & 3.539 & 1 & .060 & 1.252 \\
\hline Constante & .104 & .301 & .119 & 1 & .730 & 1.109 \\
\hline
\end{tabular}

Tabla 10. Clasificación problemas auditivos. Fuente: SPSS

\begin{tabular}{|c|c|c|c|}
\hline \multirow{2}{*}{ Observado } & \multicolumn{3}{|c|}{ Pronosticado } \\
\cline { 2 - 4 } & $\mathrm{No}$ & $\mathrm{Si}$ & $\begin{array}{c}\text { Porcentaje } \\
\text { correcto }\end{array}$ \\
\hline $\mathrm{No}$ & 583 & 57 & $91.1 \%$ \\
\hline $\mathrm{Si}$ & 277 & 68 & $19.7 \%$ \\
\hline $\begin{array}{c}\text { Porcentaje } \\
\text { global }\end{array}$ & $87.3 \%$ & $12.7 \%$ & $66.1 \%$ \\
\hline
\end{tabular}

\section{B. Análisis con Regresión logística para problemas auditivos}

El ruido excesivo, también afecta mucho las relaciones sociales, debido a que la adecuada comunicación se ve afectada y las personas suelen reaccionar agresivamente cuando se perturba su tranquilidad. Uno de los efectos es el tener que aumentar el volumen de la voz con lo que la plática cambia de tono y muchas veces de sentido (tabla 11).

Tabla 11. Clasificación problemas auditivos. Fuente: SPSS

\begin{tabular}{|c|c|c|c|}
\hline \multirow{2}{*}{ Observado } & \multicolumn{3}{|c|}{ Pronosticado } \\
\cline { 2 - 4 } & No & $\mathrm{Si}$ & $\begin{array}{c}\text { Porcentaje } \\
\text { correcto }\end{array}$ \\
\hline No & 583 & 57 & $91.1 \%$ \\
\hline $\mathrm{Si}$ & 277 & 68 & $19.7 \%$ \\
\hline $\begin{array}{c}\text { Porcentaje } \\
\text { global }\end{array}$ & $87.3 \%$ & $12.7 \%$ & $66.1 \%$ \\
\hline
\end{tabular}




\subsubsection{Análisis para problemas respiratorios} Se reunieron las variables que tuvieron una mayor incidencia en los problemas respiratorios, de acuerdo con los resultados obtenidos mediante los métodos árboles de clasificación y regresión logística.

A. Análisis con árboles de clasificación para problemas respiratorios

Al realizar el árbol de clasificación se observa que en los problemas respiratorios las variables que tienen una mayor incidencia son la práctica de algún deporte, el estado civil, la secreción nasal, la ronquera y la edad. Según NIOSH (instituto nacional para la seguridad y salud ocupacional) los trabajadores expuestos a las emanaciones del asfalto pueden experimentar efectos en la salud como: dolor de cabeza, irritación de la piel, ojos, nariz y garganta, náusea, fatiga y mareo (tabla 12 y 13).

Tabla 12. Variables en la ecuación problemas respiratorios.

Fuente: SPSS

\begin{tabular}{|l|c|c|c|c|c|c|}
\hline & B & E.T. & Wald & gl & Sig. & Exp(B) \\
\hline Deporte* $^{*}, 394$ &, 155 & 6,464 & 1 &, 011 & 1,483 \\
\hline $\begin{array}{l}\text { Otros } \\
\text { síntomas* }\end{array}$ &,- 940 &, 378 & 6,166 & 1 &, 013 &, 391 \\
\hline $\begin{array}{l}\text { Secreción } \\
\text { nasal* }\end{array}$ &, 179 &, 086 & 4,349 & 1 &, 037 & 1,196 \\
\hline Ronquera* &, 198 &, 103 & 3,692 & 1 &, 055 & 1,219 \\
\hline $\begin{array}{l}\text { Estado } \\
\text { civil* }\end{array}$ &,- 246 &, 147 & 2,815 & 1 &, 093 &, 782 \\
\hline Edad &, 003 &, 065 &, 002 & 1 &, 963 & 1,003 \\
\hline $\begin{array}{l}\text { Dolor en } \\
\text { manos }\end{array}$ &, 044 &, 068 &, 415 & 1 &, 519 & 1,045 \\
\hline
\end{tabular}

Tabla 13. Clasificación - problemas respiratorios. Fuente: SPSS

\begin{tabular}{|c|c|c|c|}
\hline \multirow{2}{*}{ Observado } & \multicolumn{3}{|c|}{ Pronosticado } \\
\cline { 2 - 4 } & No & $\mathrm{Si}$ & $\begin{array}{c}\text { Porcentaje } \\
\text { correcto }\end{array}$ \\
\hline $\mathrm{No}$ & 625 & 42 & $93,7 \%$ \\
\hline $\mathrm{Si}$ & 272 & 46 & $14,5 \%$ \\
\hline $\begin{array}{c}\text { Porcentaje } \\
\text { global }\end{array}$ & $91,1 \%$ & $8,9 \%$ & $68,1 \%$ \\
\hline
\end{tabular}

B. Análisis con regresión logística para problemas respiratorios

$\mathrm{Al}$ realizar la regresión logística se observa que en los problemas respiratorios las variables que tienen una mayor incidencia son la práctica de algún deporte, otros síntomas, la secreción nasal, la ronquera y el estado civil.

Según algunos estudios, los trabajadores también experimentan un mayor riesgo de contraer cáncer pulmonar. En vista de este posible riesgo para la salud y de los efectos relacionados con la irritación causada por el trabajo con asfalto caliente, tiene sentido tomar las medidas necesarias para controlar las exposiciones a estos riesgos relacionados con la salud [13].

Tabla 14. Tabla de clasificación problemas respiratorios. Fuente: SPSS

\begin{tabular}{|c|c|c|c|}
\hline \multirow{3}{*}{ Observado } & \multicolumn{3}{|c|}{ Pronosticado } \\
\hline & \multicolumn{2}{|c|}{ Dolor de espalda } & \multirow{2}{*}{$\begin{array}{c}\% \\
\text { Correcto }\end{array}$} \\
\hline & No & $\mathrm{Si}$ & \\
\hline No & 650 & 17 & 97,5 \\
\hline $\mathrm{Si}$ & 301 & 17 & 5,3 \\
\hline$\%$ Global & & & 67,7 \\
\hline
\end{tabular}

\subsection{Análisis con tablas de contingencia de la lesión o daño aparente y parte del cuerpo afectada}

Al comparar los resultados obtenidos del análisis de los accidentes de trabajo con el tipo de lesión o daño aparente se observa que las lesiones más frecuentes son heridas, raspaduras, golpes, fracturas, esguinces, luxaciones e insolaciones, las cuales tiene una clara relación con las partes del cuerpo más afectadas por los accidentes las cuales son las extremidades inferiores y extremidades superiores (Fig. 1, Fig. 2).

La realización de tareas, con un escalafón inferior a la capacidad del individuo, es un claro factor de insatisfacción, que provoca frustración personal y profesional [14]. 


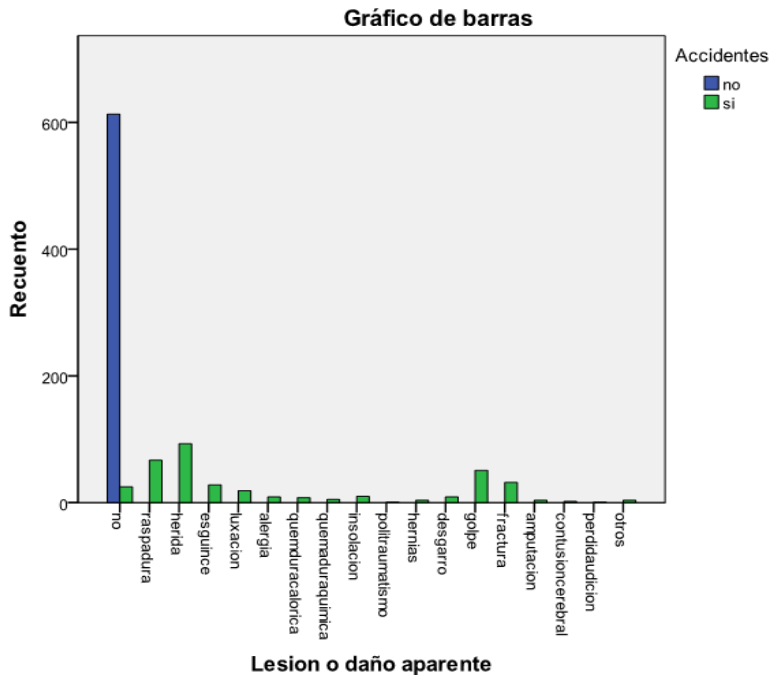

Fig. 1. Lesión o daño aparente. Fuente: SPSS

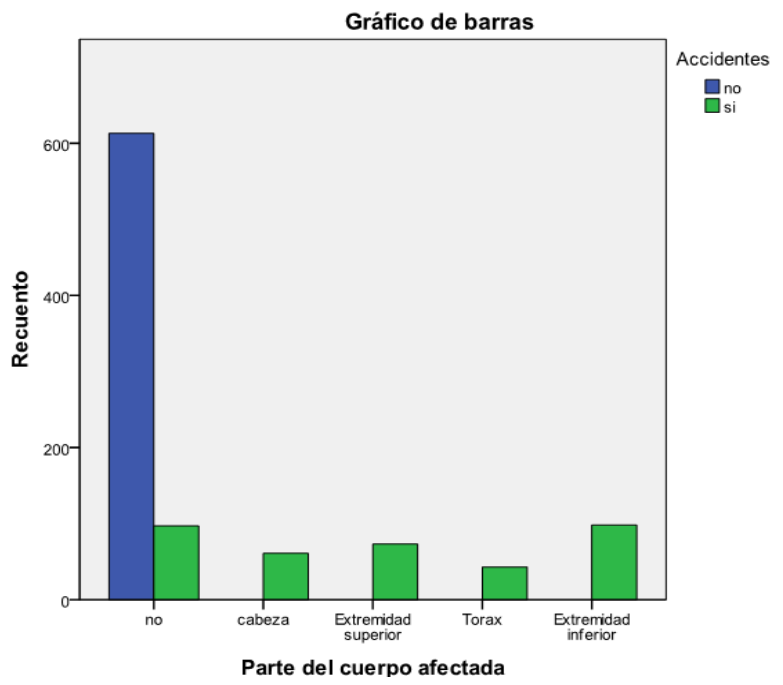

Fig. 2. Parte del cuerpo afectada. Fuente: SPSS

Esto se explica porque los riesgos existentes en la industria de la construcción y las condiciones generales en las obras son tales que, pese a las medidas preventivas que recomiende el área de salud ocupacional para la realización de las tareas, se hace necesario el uso de equipo de protección personal (EPP), sin embargo, el uso de EPP presenta la desventaja de que el personal tiene tendencia a no usarlos y entre los riesgos que se generan normalmente de este tipo de actividad están: golpes contra objetos, caída de objetos, derrame o salpicadura de líquidos calientes o corrosivos, lesiones de los pies como las causadas por la penetración de objetos (clavos en la planta del pie), y las debidas a aplastamiento del pie por materiales que caen. En esta actividad, las manos y muñecas sufren más lesiones que ninguna otra parte del cuerpo, como heridas abiertas, raspaduras, fracturas, luxaciones, esguinces, amputaciones, quemaduras, etc. que en su mayoría se pueden evitar con el uso de elementos de protección personal adecuados.

A veces los obreros conocen los riesgos y sus consecuencias, pero no utilizan protección, argumentando que el equipo es incómodo o no está a mano cuando lo necesitan. Un alto porcentaje de lesiones puede prevenirse usando elementos de protección personal.

\section{CONCLUSIONES}

Una vez realizado todo el análisis estadístico a partir de tablas de contingencia se encontró:

Del análisis de árboles se desprende que las investigaciones adelantadas por los autores y consignadas en el diseño de la encuesta, sigue siendo más importante para la accidentalidad de los trabajadores del sector de pavimento con asfaltos, la escolaridad y la destreza física revelada en las prácticas deportivas y que las mismas relaciones existentes del trabajador con sus seres más cercanos también afectan significativamente su concentración y que a la postre le significaran accidentes de trabajo. Es posible que el nivel de formación conduzca a mayor concentración como lo sugieren investigaciones previas, mientras que la aplicación de prácticas deportivas pueden dar habilidades y disciplina a la hora de hacer trabajos físicos como lo exige esta tipología laboral. Curiosamente a nivel estadístico se refleja que es factor incidente para la accidentalidad la mala relación con sus hijos que con sus esposas lo que en realidad puede sugerir el gran papel que juega los factores psicológicos y emocionales en el trabajador lo quelo conllevaa una desconcentración adicional y conduce al error. Un tema para seguir con futuras investigaciones es el de la incidencia 
del cigarrillo con la causa de accidentes, aunque es posible que ello tenga una relación indirecta con el mismo estado físico que como se demostró si influye en la accidentalidad. Un tema para seguir con futuras investigaciones es el de la incidencia del cigarrillo con la causa de accidentes, aunque es posible que ello tenga una relación indirecta con el mismo estado físico que como se demostró si influye en la accidentalidad.

Descriptivamente se muestra que de los 372 trabajadores que han sufrido accidentes de trabajo, del análisis estadístico expuesto anteriormente se destaca que las principales variables el $88.44 \%$ corresponden a la categoría de educación básica (no escolarizado, primaria y bachillerato) y el $11.56 \%$ corresponde a la categoría de educación superior (tecnólogo, universitario, posgrado y otros), así mismo el $49.39 \%$ ha sufrido accidentes de trabajo y de los que no practican deporte el $66.93 \%$ ha sufrido accidentes de trabajo, por otro lado, el $40.59 \%$ han sufrido accidentes de trabajo y de los que no fuman el $59.40 \%$ ha sufrido accidentes de trabajo, finalmente Los trabajadores que han sufrido accidentes de trabajo y que tienen hijos el $72.58 \%$ han sufrido accidentes, mientras que los que no tienen hijos el $27.42 \%$ han sufrido accidentes de trabajo.

Según el análisis realizado mediante las tablas de contingencia, en los 365 trabajadores a los cuales se les ha diagnosticado problemas de espalda se encontró lo siguiente:

$34,79 \%$ ha presentado síntomas de dolor de espalda ocasionalmente, $28,21 \%$ no presenta síntomas de dolor de espalda, 20,8\%2 presenta síntomas de dolor de espalda frecuentemente y $16,18 \%$ rara vez ha presentado estos síntomas, lo cual indica que el $71,79 \%$ de los trabajadores que han sufrido accidentes de trabajo y a los cuales se las ha diagnosticado problemas de espalda presentan síntomas de dolor de espalda. Por otro lado, 54,24\% no presenta síntomas de dolor en la manos, $18,08 \%$ ha presentado dolor en la manos de manera ocasional, 16,98 ha presentado dolor en la manos frecuentemente y $10,7 \%$ rara vez ha presentado estos síntomas, lo cual indica que el $45,76 \%$ de los trabajadores que han sufrido accidentes de trabajo y a los cuales se las ha diagnosticado problemas de espalda presentan síntomas de dolor en las manos. El 80\% corresponde a trabajadores de un nivel operativo (auxiliares y operadores), mientras el $20 \%$ restantes corresponde al nivel de supervisión (residentes, inspectores, topografía), lo cual indica que el $80 \%$ de los trabajadores que han sufrido accidentes de trabajo y a los cuales se las ha diagnosticado problemas de espalda se encuentran dentro del nivel operativo. $86.84 \%$ no ha presentado problemas de depresión, mientras que el $13,16 \%$ ha presentado problemas de depresión, lo cual indica que el 13,16\% de los trabajadores que han sufrido accidentes de trabajo y a los cuales se las ha diagnosticado problemas de espalda en algún momento han presentado problemas de depresión. Un buen asiento es aquel que ayuda a quien se sienta en él a estabilizar las articulaciones de su cuerpo, de manera que pueda mantenerse en una postura confortable [10].

Es claro que para la labor de pavimentación se requiere de grandes esfuerzos físicos y que de una u otra desencadenan problemas en la espalda, que a la postre desencadenan accidentes laborales, esto debe ser una alerta temprana para evitarlos en tanto que mientras el trabajador manifieste dolores en la espalda se debe hacer acciones correctivas y prestar mayor atención por parte de los supervisores del trabajo, Pero más interesante resulta la influencia de los problemas en manos con la generación de accidentes, sin lugar a dudas que este factor provoca menos accidentes que los dolores de espalda si resulta interesante también como alerta temprana saber que para el caso de los trabajadores de pavimentos como dolencias en manos a al postre desencadenaran accidentes.

Una vez analizada la información con tablas de contingencia para los 345 trabajadores a los cuales se les ha diagnosticado problemas auditivos por parte de un medico se encontró: El 89,85\% de los trabajadores que han sufrido accidentes de trabajo y a los cuales se las ha 
diagnosticado problemas auditivos mantienen buenas relaciones con sus compañeros de trabajo. También el $85.79 \%$ no ha presentado problemas dedepresión, mientras que el 14,21\% ha presentado problemas de depresión, lo cual indica que el $14,21 \%$ de los trabajadores que han sufrido accidentes de trabajo y a los cuales se las ha diagnosticado problemas auditivos en algún momento han presentado problemas de depresión. El 67,82\% no presenta síntomas de disminución de la audición, el 13,91\% ha presentado síntomas de disminución de la audición con frecuencia, el 9,27\% rara vez presenta síntomas de disminución de la audición y el $9 \%$ ha presentado estos síntomas ocasionalmente, lo cual indica que el 32,18\% de los trabajadores que han sufrido accidentes de trabajo y a los cuales se las ha diagnosticado problemas auditivos presentan síntomas de disminución de la audición. De estos el $81,15 \%$ corresponde a trabajadores de un nivel operativo (auxiliares y operadores), mientras que el 18,85\% restantes corresponde al nivel de supervisión (Residentes, inspectores, topografía), lo cual indica que el $81,85 \%$ de los trabajadores que han sufrido accidentes de trabajo y a los cuales se las ha diagnosticado problemas auditivos se encuentran dentro del nivel operativo. Finalmente el 77,97\% no presenta síntomas de problemas de memoria, el $15,07 \%$ rara vez presenta síntomas de problemas de memoria, el 5,50\% ha presentado estos síntomas ocasionalmente y el 1,46\% ha presentado estos síntomas con frecuencia, lo cual indica que el $22,03 \%$ de los trabajadores que han sufrido accidentes de trabajo y a los cuales se las ha diagnosticado problemas auditivos presentan síntomas de problemas de memoria.

Es claro que estas actividades desencadenan problemas auditivos por la maquinaria y las herramientas utilizadas, y no menos cierto es estas disminuciones y dolencias auditivas de una u otra manera afectan la concentración del colaborador lo que a su vez hacen que la accidentalidad se aumente significativamente, así si mismo se confirma que no solo es la exposición a estas fuentes de emisión de ruidos sino el tiempo de exposición es por ello que los trabajadores de mayor tiempo de exposición como operario tengan mayores problemas auditivos $\mathrm{y}$ por ende mas posibilidad de accidentes que aquellos como ingenieros $\mathrm{y}$ topógrafos que si bien son expuestos a los mismos ruidos el tiempo de permanencia a la fuente de emisión es menor. Importante para este nivel de investigación ha sido como con el análisis de arboles se demuestra una incidencia (que si bien no es la más alta) de problemas auditivos con la memoria, que amerita estudios específicos si puede significar falencias en los niveles de concentración.

Al realizar el análisis con tablas de contingencia a los 318 trabajadores a los cuales se les ha diagnosticado problemas respiratorios por parte de un medico se encontró:

$67,61 \%$ corresponde a trabajadores que no practican deporte, mientras que el 32,29\% restantes corresponde a trabajadores que practican algún deporte, lo cual indica que el $67,61 \%$ de los trabajadores que han sufrido accidentes de trabajo y a los cuales se las ha diagnosticado problemas respiratorios no practican deporte. También se determino que el $64,77 \%$ no presenta secreción nasal, el 15,09\% ocasionalmente ha presentado secreción nasal, el 14,46\% rara vez ha presentado secreción nasal y el 5,68\% frecuentemente ha presentado secreción nasal, lo cual indica que el 35,23\% de los trabajadores que han sufrido accidentes de trabajo y a los cuales se las ha diagnosticado problemas respiratorios han presentado síntomas de secreción nasal. El 70,75\% no presenta ronquera, el $16,03 \%$ rara vez ha presentado ronquera, el 8,49\% ocasionalmente ha presentado secreción nasal y el 4,73\% frecuentemente ha presentado secreción nasal, lo cual indica que el $29,25 \%$ de los trabajadores que han sufrido accidentes de trabajo y a los cuales se las ha diagnosticado problemas respiratorios han presentado síntomas de ronquera. De estos el $42,76 \%$ corresponde a trabajadores cuyo estado civil es solo, mientras que el $57,24 \%$ restantes corresponde a trabajadores casados o en unión libre, lo cual indica que el $57,24 \%$ de los trabajadores que han sufrido accidentes de trabajo y a los cuales 
se las ha diagnosticado problemas respiratorios son casados o en unión libre.

Es claro y sabido que las actividades de pavimentación con asfaltos tenga una gran influencia negativa sobre los problemas respiratorios que quedan plenamente corroboradas con las anteriores cifras descriptivas. Muy curioso resulta la correlación que refleja el análisis de arboles de los problemas respiratorios relacionados con

\section{Referencias Bibliográficas}

[1] A. Cáceres, C. Luis; Legislación en salud ocupacional y riesgos profesionales. Bogotá: Salud Laboral, 2005.

[2] ARSEG. Compendio de Normas Legales Sobre Salud Ocupacional, 2006.

[4] K. Ringen, J. Seegal, L. James, Enciclopedia de salud y seguridad en el trabajo - Volumen 3 - Capitulo 93 (Construcción), 1998.

[5] IARC (International Agency for Research on Cancer), [En línea], consultado en Julio 10 del 2011, disponible en: http:// www.iarc.fr/

[6] C. Vinelo, Técnicas de Muestreo, Editorial Pueblo y Educación, La Habana Cuba, 1978.

[7] A. Joann, A. Wess, D. Larry, M. HARING, Química concisa: la evaluación internacional de asfalto. Instituto Nacional de Seguridad y Salud Laboral, Cincinnati, Ohio, EE.UU, 2007. el estado civil de los trabajadores, ya que en apariencia no debería existir este tipo de relación y de haberlo debería ser al contrario, es posible explicarlos frente a otras variables como la edad, es decir que si bien es cierto los problemas respiratorios aumentan conforme pasan los años es posible inferir que a mas edad también el estado civil cambia de soltería a un estado de tener una relación formal o informal es decir trabajadores casados o en unión libre.

[8] S. Loyo, Seguridad en el manejo de materiales asfálticos, Control de calidad, Empresa Daycoven, 2007.

[9] D. Peña, Análisis de datos multivariantes, Editorial Mc Graw Hill, 2002.

[10] L. Breiman, J. Friedman, R. Olshen, P. Stone, Classication and regression trees, Wadsworth International Group, 1984.

[11] SPSS Classification Trees ${ }^{\mathrm{TM}}$ 13.0, Editorial SPSS Inc, Irlanda, 2004.

[12] N. Freivalds, Ingeniería Industrial: Métodos, estándares y diseño del trabajo, Décimo Primera edición, Editorial Alfa Omega, 2004.

[13] Fundación MAPFRE, Manual de higiene Industrial, 1991.

[14] F. Alonso, Aspectos psicosociales de la prevención de riesgos en el trabajo en la actualidad Laboral, D.C: Legis, No. 19, 1987, Bogotá.

[15] D. Oborne, Ergonomía en acción: La Adaptación del medio de trabajo al hombre. Editorial: Trillas, 1987. 